\title{
Short-term poor glycemic control and retinal microvascular changes in pediatric Type 1 Diabetes patients in Singapore: a pilot study
}

Ling-Jun $\mathrm{Li}^{1,2^{*}}$, Ecosse Lamoureux ${ }^{1,2}$, Tien Yin Wong ${ }^{1,2}$ and Ngee Lek ${ }^{2,3}$

\begin{abstract}
Background: Poor glycemic control in Type 1 Diabetes (T1D) patients is strongly associated with an increased risk of diabetes-related microvascular complications later in life, but it is unclear whether short period of poor glycemic control in children with T1D can cause evident microvascular morphological changes long before any pathological manifestation. Our study aimed to investigate the longitudinal association between poor glycemic control and subsequent changes in retinal microvasculature, in a pilot study of 55 pediatric T1D patients from Singapore after a one-year follow-up.
\end{abstract}

Methods: This is a hospital-based, exposure-matched and retrospective longitudinal study. A total of 55 T1D patients were included from Singapore KK Women's and Children Hospital, 28 of whom had poor glycemic control (average glycated hemoglobin $[\mathrm{HbA} 1 \mathrm{c}] \geq 8 \%$ during the year) while the other 27 age- and gender-matched subjects had good glycemic control (HbA1c $<8 \%)$. Retinal photography was taken at diabetes annual screening and images were graded by trained graders using a semi-automated computer-based program (Singapore I Vessel Assessment [SIVA], version 4.0, Singapore Eye Research Institute, Singapore) and a spectrum of retinal vascular parameters (e.g. caliber, tortuosity, branching angle and fractal dimension) were measured quantitatively from 0.5 to 2.0 disc diameters.

Results: There was no significant difference in ethnicity, duration of T1D, blood pressure, body mass index (BMI) and low-density cholesterol lipoprotein (LDL) between the two groups. Retinal imaging was obtained at the end of 1 year of glycemic control assessment. In multiple linear regression adjusting for ethnicity, BMI, LDL and duration of T1D, patients with poor glycemic control tended to have marginally wider retinal arteriolar caliber $(6.0 \mu \mathrm{m}, 95 \% \mathrm{Cl}$ : $-0.9,12.8)$ and had significantly larger retinal arteriolar branching angle (10.1 degrees, 95\% Cl: 1.4, 18.9) compared with their age- and gender- matched counterparts with good glycemic control.

Conclusions: Our findings showed that abnormal retinal microvascular morphology was evident in pediatric patients with T1D after one-year's poor glycemic control. Such morphological abnormalities may lead to future development of microvascular complications among T1D pediatric patients with poor glycemic control.

Keywords: Glycemic control, Retinal microvascular changes, Type 1 Diabetes (T1D), Children

\footnotetext{
* Correspondence: queenie.li.l.j@seri.com.sg

${ }^{1}$ Singapore Eye Research Institute, Singapore National Eye Centre, Singapore,

Singapore

2Duke-NUS Medical School, Department of Pediatrics, Singapore, Singapore

Full list of author information is available at the end of the article
}

\section{Ciomed Central}

(c) The Author(s). 2017 Open Access This article is distributed under the terms of the Creative Commons Attribution 4.0 International License (http://creativecommons.org/licenses/by/4.0/), which permits unrestricted use, distribution, and reproduction in any medium, provided you give appropriate credit to the original author(s) and the source, provide a link to the Creative Commons license, and indicate if changes were made. The Creative Commons Public Domain Dedication waiver (http://creativecommons.org/publicdomain/zero/1.0/) applies to the data made available in this article, unless otherwise stated. 


\section{Background}

Type 1 Diabetes (T1D) is a chronic disease characterized by elevated blood glucose which results from lack of endogenous insulin subsequent to autoimmune destruction of pancreatic beta cells [1]. Hyperglycemia is known to be associated with increased shear stress and impaired microvascular endothelium [2] which leads to microvascular complications such as retinopathy, neuropathy and nephropathy [3]. Following diagnosis of T1D in young subjects, optimal glycemic control is strongly recommended as it is associated with a reduced risk of diabetes-related complications later in life [4].

Substantial evidence has shown that retinal imaging is a non-invasive, reproducible and reliable technology to view small vessel morphology, and changes in these microvascular features can reflect the degree of endothelial dysfunction in T1D patients [3]. In several crosssectional population-based studies, retinal arteriolar and venular widening, increased retinal arteriolar tortuosity and enlarged retinal arteriolar branching angle were associated with increased concentrations of fasting plasma glucose (FPG) and glycated haemoglobin (HbA1C), as well as a longer duration of T1D both in children and adults [5, 6]. However, it is unclear whether such changes are already evident after a short period of poor diabetes control in children with T1D.

As poor glycemic control may cause endothelial dysfunction in vivo, and can be reflected in real-time retinal microvascular changes, we investigated the longitudinal association between glycemic control and subsequent changes in retinal microvasculature, in a pilot study of 55 pediatric T1D patients from Singapore after a oneyear follow-up. We hypothesized that short-term poor glycemic control is associated with suboptimal retinal vascular morphology in pediatric patients with T1D.

\section{Methods}

\section{Study design}

This is a hospital-based, exposure matched and retrospective longitudinal study. Twenty-eight pediatric T1D patients aged 10-16 years with poor glycemic control and 27 age- and gender- matched pediatric T1D patients with good glycemic control who attended follow-up outpatient appointments in the diabetes clinical service at KK Women's and Children's hospital (KKH) in Singapore, were included in the present analyses. All the patients were on insulin replacement treatment and were not complicated by any degree of diabetic retinopathy (DR). Eligible subjects were Singapore citizens or long-term residents, who had quarterly HbA1C measurements and annual retinal screening test according to the American Diabetes Association (ADA) 2016 guidelines (http://www.ndei.org/ADA-diabetes-managementguidelines-microvascular-complications-neuropathy- retinopathy-nephropathy-diabetic-kidney-diseasefootcare.aspx.html). This study was approved by SingHealth Centralized Institutional Review Board, and was conducted according to the tenets of the Declaration of Helsinki.

\section{Exposure-Glycemic control}

Venous whole blood specimens were collected in EDTA tubes at each 3-monthly clinic visit. HbA1C was measured using the Cobas Integra 800 (Roche Diagnostics, Rotkreuz, Switzerland), based on the turbidimetric inhibition immunoassay for hemolyzed whole blood (reference range 4.4-6.4\%). HbA1C measurements were standardized to the reference method aligned with the Diabetes Control and Complications Trial and the American National Glycohemoglobin Standardization Program standards [7]. The intra-assay coefficient of variation was $2.3 \%$ and the inter-assay coefficient of variation was $2.4 \%$. Since venous whole blood specimens were collected in EDTA tubes at a quarterly clinic visit, we calculated average $\mathrm{HbA} 1 \mathrm{C}$ at a yearly basis with four readings. For the purpose of the present analyses, good glycemic control was defined as an average $\mathrm{HbA} 1 \mathrm{C}$ value of less than $8 \%$ within a year, while poor glycemic control was defined as $8 \%$ and above [8].

\section{Outcome-Retinal microvascular assessment}

Retinal photography was performed at the year-end clinic visit. Right eye digital retinal photographs were taken from participants using a $45^{\circ}$ non-mydriatic retinal camera (Canon CR-1, 40D SLR digital retinal camera backing; Canon Inc., Japan) after pharmacological pupil dilation. Two retinal images centered on the optic disc and macular were taken. Retinal photographs were assessed by trained graders using a semi-automated computer-based program (Singapore I Vessel Assessment [SIVA], version 4.0, Singapore Eye Research Institute, Singapore) and a spectrum of retinal vascular parameters were measured quantitatively from 0.5 to 2.0 disc diameters (zone $\mathrm{C}$ ) away from the optic disc margin. [9] Retinal vascular caliber, branching angle, tortuosity and fractal dimension were assessed according to standard protocol, which have been described in detail in previous publications [9]. Intra-grader reliability was assessed in $10 \%(n=6)$ of randomly selected retinal photographs from our study. The intra-class correlation coefficient (ICC) was above 0.90 for all retinal vascular parameters.

\section{Co-variates}

Data on duration of $\mathrm{T} 1 \mathrm{D}$, ethnicity, parental education and housing condition were collected at the baseline visit. Patients' weight, height, systolic (SBP) and diastolic (DBP) blood pressure were measured at baseline visit, 
and type of T1D medication, pubertal stage, foot review and dietician review were evaluated at diabetic screening clinic at each follow-up visit. Body mass index (BMI) was calculated as weight $(\mathrm{kg})$ divided by square of height $\left(\mathrm{m}^{2}\right)$. Fasting glucose, cholesterol, high-density cholesterol lipid (HDL), low-density cholesterol lipid (LDL), triglyceride, free thyroxine (T4), thyroid stimulating hormone and urine albumin/creatinine ratio (uACR) were examined at each visit.

\section{Statistical analysis}

Glycemic control (good vs. poor) was analysed as a dichotomous variable. Retinal microvascular measures were normally distributed and analysed as continuous variables. Comparisons of baseline variables were examined using a 2-sample independent student t-test (for continuous variables), $x^{2}$ test or fisher exact t-test (for categorical variables).

Multiple linear regression was used to analyse the association between one-year glycemic control (poor vs. good) and retinal microvascular measures. Since this is an exposure-matched cohort, four models were tested: Model 1-unadjusted; Model 2-adjusted for ethnicity; Model 3-Model 2 and additionally adjusted for baseline BMI and LDL; Model 4-Model 3 and additionally adjusted for duration of T1D. Pubertal stage and uACR were also included into the fully-adjusted model in a sensitivity analysis. Mean difference in estimates and 95\% confidence interval were described. All regression analyses were performed using SPSS 19.0 (SPSS Inc., Chicago, U.S.). Potential effect modifications by ethnicity and child sex were examined by including multiplicative terms with glycemic control (HbA1C*ethnicity, HbA1C*child sex) into the fully-adjusted model.

\section{Results}

Comparisons of baseline variables between subjects with good $(n=27)$ and poor glycemic control $(n=28)$ are described in Table 1. No significant differences were found between both groups in terms of age, gender, ethnicity, duration of T1D, SBP, DBP, BMI, LDL and uACR at baseline. Compared to subjects with good glycemic control, those with poor glycemic control had wider retinal arteriolar caliber (139.84 vs. $133.81 \mu \mathrm{m}, p=0.03$ ) and larger retinal arteriolar branching angles (85.76 vs. 78.28 degree, $p=0.04$ ).

After adjusting for ethnicity, baseline BMI and LDL and duration of T1D, pediatric patients with one-year poor glycemic control tended to have marginally wider retinal arteriolar caliber $(6.0 \mu \mathrm{m}, 95 \% \mathrm{CI}$ : $-0.9,12.8)$ and significantly larger retinal arteriolar branching angle (10.1 degree, 95\% CI: 1.4, 18.9), compared with their age- and gender- matched counterparts with good glycemic control (Table 2). Retinal arteriolar tortuosity, fractal dimension and all venular parameters however, were not associated with poor glycemic control. No potential effect modifications by ethnicity or child sex were observed.

Figure 1 showed the difference in retinal arteriolar caliber and retinal arteriolar branching angle between T1D patients, one of which had 1-year good glycemic control while the other had one-year poor glycemic control.

\section{Discussion}

In this pilot study, we found that short-term poor glycemic control was associated with wider retinal arteriolar caliber and larger retinal arteriolar branching angle in a sample of pediatric T1D patients in Singapore.

Owing to the non-invasive and reproducible nature of the retinal microvasculature imaging, changes in retinal vascular geometry have been extensively studied and validated as proxies for small-vessel dysfunction, particularly in diabetes and other microvascular disorders [3]. We found that short-term poor glycemic control was associated with suboptimal retinal microvascular morphology including wider retinal arteriolar caliber and larger retinal arteriolar branching angle. Small-vessel dysfunction has been shown to be a consequence of T1D, possibly as a result of endothelial dysfunction and inflammation resulting from chronic hyperglycemia [10, 11]. Increased vascular shear stress and impaired microvascular endothelium mediated by hyperglycemia has been demonstrated in diabetic patients, and chronic hyperglycemia has been proven to lead to vasodilation of retinal arterioles and venules [12, 13]. Furthermore, a larger branching angle would increase the workload and energy spent, and thereby reducing the efficiency of retinal blood flow [14]. Such alterations in branching angle have been correlated with atherosclerosis, deranged blood flow and endothelial damage [15]. In our study, we found that both wider caliber and enlarged branching angle in retinal arterioles was associated with higher HbA1C episodes in the past year. Such morphological abnormalities may indicate a worse hypoxia-induced formation and a lower complexity of less than ideal oxygenation caused by chronic hyperglycemia, which may shed light on future development of microvascular complications (i.e. retinopathy) among such poor glycemic control pediatric patients.

In this short-term longitudinal study, measurements for both exposure and outcome followed standardized protocols and were analyzed by qualified staff. This study however, is not without limitations. First, our study had a relatively small sample for analysis, which might have potential selection bias in truly representing the pediatric T1D population in Singapore. Second, the small sample size might not be sufficiently powered to detect other retinal vascular parameters in relation to poor glycemic control. Third, residual confounding (i.e. 
Table 1 Comparisons of clinical characteristics between T1D pediatric patients with good and poor glycemic control

\begin{tabular}{|c|c|c|c|}
\hline & $\begin{array}{l}\text { Good glycemic control } \\
(n=27) \\
\text { mean, SD or } n, \%\end{array}$ & $\begin{array}{l}\text { Poor glycemic control } \\
(n=28) \\
\text { mean, SD or } n, \%\end{array}$ & $p$ value $^{*}$ \\
\hline \multicolumn{4}{|l|}{ Baseline characteristics, } \\
\hline Age, years & $13.3,1.9$ & $13.0,1.8$ & 0.45 \\
\hline Sex, male & $14,51.9 \%$ & $11,39.3 \%$ & 0.35 \\
\hline \multicolumn{4}{|l|}{ Ethnicity, } \\
\hline Chinese & $20,74.1 \%$ & $13,46.4 \%$ & 0.05 \\
\hline Malay & $2,7.4 \%$ & $9,32.1 \%$ & \\
\hline Indian & $5,18.5 \%$ & $6,21.4 \%$ & \\
\hline Duration of diagnosis, years & $4.17,3.8$ & $5.64,3.1$ & 0.12 \\
\hline \multicolumn{4}{|l|}{ Anthropometric measures, } \\
\hline $\mathrm{SBP}, \mathrm{mmHg}$ & $106.2,10.8$ & $104.6,11.2$ & 0.85 \\
\hline $\mathrm{DBP}, \mathrm{mmHg}$ & $64.9,7.1$ & 61.75 .8 & 0.10 \\
\hline $\mathrm{BMI}, \mathrm{kg} / \mathrm{m}^{2}$ & $19.4,3.2$ & $20.4,2.9$ & 0.25 \\
\hline \multicolumn{4}{|l|}{ Serum biomarkers, } \\
\hline $\mathrm{LDL}, \mathrm{mmol} / \mathrm{L}$ & $2.9,0.9$ & $3.1,0.9$ & 0.49 \\
\hline $\mathrm{uACR}, \mathrm{mg} / \mathrm{g}$ & $1.4,1.8$ & $1.0,0.8$ & 0.60 \\
\hline
\end{tabular}

Retinal vascular parameters

Retinal arterioles,

$\begin{array}{llll}\text { Caliber, } \mu \mathrm{m} & 133.8,10.6 & 139.8,9.5 & 0.03 \\ \text { Fractal dimension, Df } & 1.22,0.05 & 1.21,0.06 & 0.24 \\ \text { Branching angle, D } & 78.3,14.7 & 85.8,11.8 & 0.04 \\ \text { Curvature Tortuosity, unit } & 9.1 \mathrm{E}-5,1.7 \mathrm{E}-5 & 9.8 \mathrm{E}-5,2.5 \mathrm{E}-5 & 0.27 \\ \text { Retinal venules, } & & 196.3,22.3 & 0.11 \\ \text { Caliber, } \mu \mathrm{m} & 188.2,13.1 & 1.20,0.04 & 0.11 \\ \text { Fractal dimension, Df } & 1.22,0.04 & 77.1,9.8 & 0.85 \\ \text { Branching angle, D } & 77.7,10.9 & 9.5 \mathrm{E}-5,1.5 \mathrm{E}-5 & 0.81 \\ \text { Curvature Tortuosity, unit } & 9.4 \mathrm{E}-5,1.7 \mathrm{E}-5 & \end{array}$

Table 2 Association between poor glycemic control and retinal microvascular parameters in T1D pediatric patients

\begin{tabular}{lll}
\hline & Retinal arteriolar caliber, $\mu \mathrm{m}$ Difference in $\beta(95 \% \mathrm{Cl})$ & Retinal arteriolar branching angle, degree Difference in $\beta(95 \% \mathrm{Cl})$ \\
\hline $\begin{array}{l}\text { Good glycemic control } \\
\text { Poor glycemic control }\end{array}$ & Reference & Reference \\
Model 1 & $6.0(0.6,11.5)$ & $7.5(0.3,14.7)$ \\
Model 2 & $5.3(-0.5,11.1)$ & $8.0(0.4,15.6)$ \\
Model 3 & $6.3(-0.3,12.9)$ & $9.1(0.5,17.7)$ \\
Model 4 & $6.0(-0.9,12.8)$ & $10.1(1.4,18.9)$ \\
\hline
\end{tabular}

Model 1, unadjusted.

Model 2, adjusted for ethnicity.

Model 3, Model 2 and additionally adjusted for BMI and LDL at baseline.

Model 4, Model 3 and additionally adjusted for duration of T1D diagnosis. 

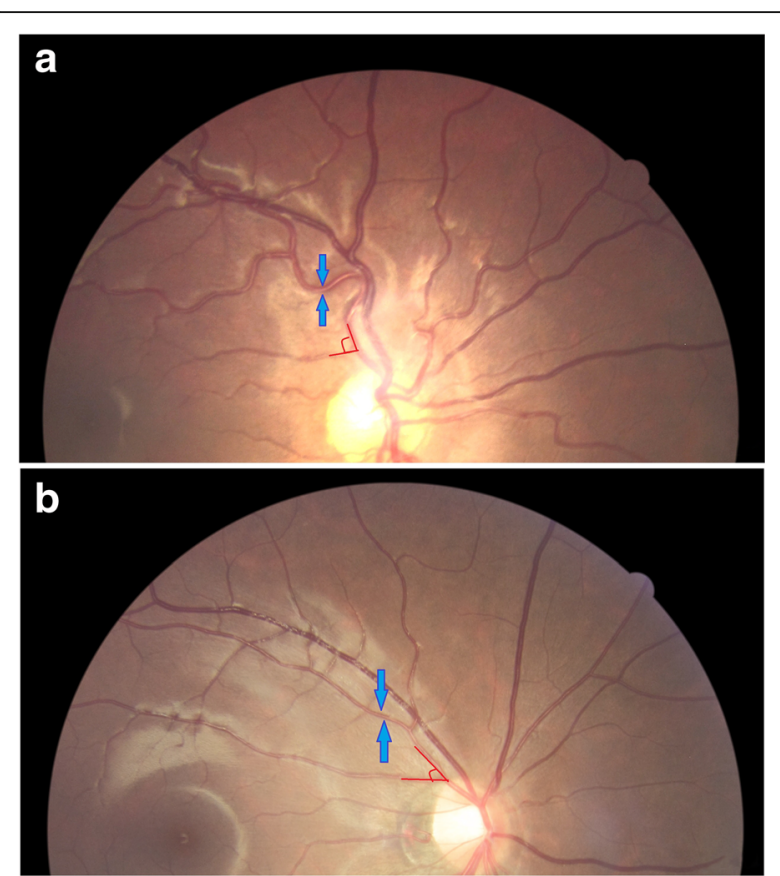

Fig. 1 Retinal images of a T1D patient with poor glycemic control and a T1D patient with good glycemic control. Comparison of retinal arteriolar caliber and retinal arteriolar branching angle between patient with poor glycemic control (a) and patient with good glycemic control (b). Blue arrows are pointing to the width of retinal arterioles while red angles are indicating retinal arteriolar branching angle. The patient with poor glycemic control had a wider average retinal arteriolar caliber (147.8 vs. $112.3 \mu \mathrm{m})$ and a larger average retinal arteriolar branching angle (110.4 vs. $\left.90.0^{\circ}\right)$ than the patient with good glycemic control

compliance to insulin treatment) due to unmeasured/uncollected confounders might potentially affect our analysis. Future studies with a larger sample size and a longer follow-up are warrant.

In conclusion, our findings showed that poor glycemic control in the past year was associated with morphological abnormalities in retinal microvasculature (wider retinal arteriolar caliber and enlarged retinal arteriolar branching angle) in a sample of Singapore T1D pediatric patients aged 10-16 years. These morphological abnormalities may indicate a worse small-vessel function caused by poor glycemic control, which may lead to future development of microvascular complications among T1D pediatric patients with suboptimal glycemic control.

\section{Conclusions}

In our hospital-based, exposure-matched and retrospective longitudinal study, a total of 55 pediatric T1D patients were included for analysis after a year's insulin treatment. Our findings showed that abnormal retinal microvascular morphology was evident in pediatric T1D patients with short-term poor glycemic control. Therefore, we suggested that morphological abnormalities of retinal microvasculature (i.e. arteriolar widening and arteriolar branching angle enlargement) may lead to future development of microvascular complications among T1D pediatric patients with poor glycemic control.

\section{Abbreviations}

T1D: Type 1 diabetes; FPG: Fasting plasma glucose; HbA1C: Glycated haemoglobin; DR: Diabetic retinopathy; ADA: American diabetes association; SIVA: Singapore I Vessel Assessment; ICC: Intra-class correlation coefficient; SBP: Systolic blood pressure; DBP: Diastolic blood pressure; BMI: Body mass index; HDL: High-density lipoprotein; LDL: Low-density lipoprotein; UACR: Urine albumin/creatinine ratio

\section{Acknowledgements}

We thank the patients and their families for participating in this study, and all clinic staff and doctors for their support in caring for these patients with T1D and for running the diabetes annual review clinics during which the retinal screening was performed.

\section{Funding}

Dr. Ling-Jun Li is funded by Singapore National Medical Research Council Transition Award (NMRC TA/0027/2014) and Singapore National Medical Research Council Research Training Fellowship (NMRC/Fellowship/0029/2014). Dr. Ngee Lek is funded by Singapore National Medical Research Council Transition Award (NMRC TA/0037/2015) and Clinician Scientist/Clinician Investigator Salary Support Programme (CS/CISSP).

\section{Availability of data and materials}

All data generated or analysed during this study are included in this published article.

\section{Authors' contributions}

L.-J. Li conducted study design, researched data and wrote manuscript; E.L. edited manuscript; T.Y.W. contributed to discussion; N.L. conducted study design and reviewed manuscript. All authors have read and approved the final manuscript.

\section{Competing interests}

The authors declare that they have no competing interests.

\section{Consent for publication}

As mentioned in the paragraph above, no consent for publication is required from individual participant.

\section{Ethics approval and consent to participate}

Eligible subjects were Singapore citizens or long-term residents, who had quarterly $\mathrm{HbA} 1 \mathrm{C}$ measurements and annual retinal screening test according to the American Diabetes Association (ADA) 2016 guidelines (http:// www.ndei.org/ADA-diabetes-management-guidelines-microvascular-complications-neuropathy-retinopathy-nephropathy-diabetic-kidney-diseasefoot-care.aspx.html). Therefore, no consent is required due to the international diabetic and retinal screening guideline. This study had ethics approval by SingHealth Centralized Institutional Review Board, and was conducted according to the tenets of the Declaration of Helsinki.

\section{Publisher's Note}

Springer Nature remains neutral with regard to jurisdictional claims in published maps and institutional affiliations.

\section{Author details}

'Singapore Eye Research Institute, Singapore National Eye Centre, Singapore, Singapore. ${ }^{2}$ Duke-NUS Medical School, Department of Pediatrics, Singapore, Singapore. 'Department of Pediatrics, KK Women's and Children's Hospital,

Singapore, Singapore. 
Received: 14 March 2017 Accepted: 22 April 2017

Published online: 15 June 2017

\section{References}

1. Broe R. Early risk stratification in pediatric type 1 diabetes. Acta Ophthalmol. 2015;93 Thesis 1:1-19.

2. Chan NN, Vallance P, Colhoun HM. Endothelium-dependent and -independent vascular dysfunction in type 1 diabetes: role of conventional risk factors, sex, and glycemic control. Arterioscler Thromb Vasc Biol. 2003;23(6):1048-54.

3. Ikram MK, Cheung CY, Lorenzi M, Klein R, Jones TL, Wong TY. Retinal vascular caliber as a biomarker for diabetes microvascular complications. Diabetes Care. 2013;36(3):750-9.

4. The effect of intensive treatment of diabetes on the development and progression of long-term complications in insulin-dependent diabetes mellitus. The Diabetes Control and Complications Trial Research Group. N Engl J Med. 1993;329(14):977-86.

5. Nguyen TT, Wang JJ, Sharrett AR, Islam FM, Klein R, Klein BE, Cotch MF, Wong TY. Relationship of retinal vascular caliber with diabetes and retinopathy: the Multi-Ethnic Study of Atherosclerosis (MESA). Diabetes Care. 2008;31(3):544-9.

6. Sasongko MB, Wong TY, Donaghue KC, Cheung N, Jenkins AJ, BenitezAguirre P, Wang JJ. Retinal arteriolar tortuosity is associated with retinopathy and early kidney dysfunction in type 1 diabetes. Am J Ophthalmol. 2012;153(1):176-83. e171

7. Oh HG, Rhee EJ. Blood pressure is the determinant for the increased risk for intracranial arterial Stenosis in subjects with elevated Glycated hemoglobin levels: the Kangbuk Samsung Health Study. J Stroke Cerebrovasc Dis. 2016;25:2729-34

8. O'Reilly JR, Reynolds RM. The risk of maternal obesity to the long-term health of the offspring. Clin Endocrinol. 2013;78(1):9-16.

9. Li LJ, Cheung CY, Ikram MK, Gluckman P, Meaney MJ, Chong YS, Kwek K, Wong TY, Saw SM. Blood pressure and retinal microvascular characteristics during pregnancy: growing up in Singapore towards healthy outcomes (GUSTO) study. Hypertension (Dallas, Tex : 1979). 2012;60(1):223-30.

10. Festa A, D'Agostino R Jr, Tracy RP, Haffner SM. Elevated levels of acutephase proteins and plasminogen activator inhibitor-1 predict the development of type 2 diabetes: the insulin resistance atherosclerosis study. Diabetes. 2002;51(4):1131-7.

11. Hu FB, Meigs JB, Li TY, Rifai N, Manson JE. Inflammatory markers and risk of developing type 2 diabetes in women. Diabetes. 2004;53(3):693-700.

12. Gonzalez JS, Tanenbaum ML, Commissariat PV. Psychosocial factors in medication adherence and diabetes self-management: implications for research and practice. Am Psychol. 2016;71(7):539-51.

13. Jeganathan VS, Sabanayagam C, Tai ES, Lee J, Lamoureux E, Sun C, Kawasaki $\mathrm{R}$, Wong TY. Retinal vascular caliber and diabetes in a multiethnic Asian population. Microcirculation (New York, NY: 1994). 2009;16(6):534-43.

14. Zamir M, Medeiros JA, Cunningham TK. Arterial bifurcations in the human retina. J Gen Physiol. 1979;74(4):537-48.

15. Stanton AV, Wasan B, Cerutti A, Ford S, Marsh R, Sever PP, Thom SA, Hughes $A D$. Vascular network changes in the retina with age and hypertension. J Hypertens. 1995;13(12 Pt 2):1724-8.

\section{Submit your next manuscript to BioMed Central and we will help you at every step:}

- We accept pre-submission inquiries

- Our selector tool helps you to find the most relevant journal

- We provide round the clock customer support

- Convenient online submission

- Thorough peer review

- Inclusion in PubMed and all major indexing services

- Maximum visibility for your research

Submit your manuscript at www.biomedcentral.com/submit 